\title{
DEVELOPMENT OF A GEODATABASE FOR EFFICIENT REMOTE SENSING DATA MANAGEMENT IN EMERGENCY SCENARIOS
}

\author{
A. Alamouri and M. Gerke \\ Technische Universität Braunschweig, Institute for Geodesy and Photogrammetry, Braunschweig, Germany \\ (a.alamouri, m.gerke)@tu-braunschweig.de
}

KEY WORDS: Emergency, Geodata, Database design, Geodata sharing, Web application

\begin{abstract}
Disasters such as floods, large fires, landslides, avalanches, or forest fires are often inevitable and cannot be fully prevented, but their impact can be minimized with sound disaster management strategies aided by the latest technological advancements. A key factor affecting these strategies is the time, because any delay can result in dramatic consequences and potentially human losses. Therefore, a quick geo-situation report of the disaster is highly demanded, but still not an easy task because - in most cases - a priori known spatial information like map data or geodatabases, are outdated, and anyway won't provide an overview on the current situation. This paper provides an exploratory investigation to be smart in providing correct and timely geodata that can help in emergency cases; especially in support decision making in emergency and risk management. In particular, issues related to geodatabase design and visualization of a variety of geodata available play a key role when it comes to efficient data deployment and usability. To this end, a significant part of this research will be devoted to develop a concept for a geodatabase design and dataset management that helps assessing a disaster risk through a potential provision of data needed. Based on this consideration, the proposed concept is to create multi-disciplinary integrated geodatabases as well as an easy-to-use graphical user interface to access the obtained data. To address this concept, hard- and software solutions are being developed through the joint research project ANKommEn and its extension ANKommEn2. In those projects two automated unmanned systems, that is an aerial UAV (Unmanned Aerial Vehicle) and a ground based UGV (Unmanned Ground Vehicle), are being developed to provide up-to-date information of rescue scenarios. Within this paper, highlights about the two project parts will be briefly presented, and then the current state of the art in geospatial database management, followed by focusing on Postgres-based database management connected with QGIS, and finally current results like a Web Map Service will be discussed.
\end{abstract}

\section{INTRODUCTON}

The project ANKommEn (german acronym for Automated Navigation and Communication for Exploration) is a research project funded by the Ministry of Economic affairs and energy (BMWI). The partners in the first project part were the Institute of Flight Guidance (IFF), the Institute of Mobile Machines and Commercial Vehicles (IMN) - both Technische Universitaet Braunschweig - and the AirRobot ${ }^{\circledR} \mathrm{GmbH} \& \mathrm{Co}$. KG, a german manufacturer of multirotor UAVs. Additionally, the professional fire brigade of Braunschweig and the NLWKN (Lower Saxony Water Management, Coastal Defense and Nature Conservation Agency) participated as associated project partners. The main result of the first project part is that an automated exploration system based on ground- and airbased unmanned vehicles has been developed (Batzdorfer, et al., 2018). The system will be implemented for the management of emergency incidents to provide up-to-date information of the situation, especially geodata like georeferenced orthophotos and point clouds. In the system, two approaches for data collection and information creation are used: an online visual Simultaneous Localization and Mapping (SLAM) and a photogrammetry pipeline using the Agisoft Photoscan API (Bobbe et al., 2017).

In every moment during emergencies, the situation can rapidly change and therefore a large amount of geo-datasets containing great number of files and object data should be respected, stored and analysed with such a multi sensor - multi platform system as implemented in the project. In such environment, a key challenge is to provide the needed spatial and also non-spatial data in an easy way based on accurate and simple solutions for an identification and retrieval of, often distributed and heterogeneous, geodata. From this aspect, considering creation of geodatabases is essential, but the design of a geodatabase is a critical stage in ensuring its effectiveness and proficiency. If a geodatabase is designed inappropriately, the data provision will not function to its full potential, essentially creating problems for the users (English, 2008). Therefore, in the second project part ANKommEn2, we concentrate on the design of geodatabases with respect to pertaining technical functionalities. The main priority is to design a proficient geodatabase concept based on the needs and responsibilities of the resource specialists and managers working in the emergency sector. The intended geodatabases should be well designed to ultimately help document, organize, edit, manage and analyse the geospatial data available. They will include not only original datasets such as images, but also derived data such as orthophotos, point clouds, metadata such as waypoints, etc. Furthermore the extension of the photogrammetric components like integration with InfraRed (IR) cameras as co-registration data, derivation of precise elevation models for orthoprojection, etc. are important datasets that should be considered in a geodatabase design. Once having a complete geodatabase, it should address needs for data provision to end users in an easy mechanism like web application that enables users/operators getting their work done without having to learn a lot of skills or technical issues. Therefore, a framework for geodata deployment and web based application is being developed. 


\section{RELATED WORK}

In general, information support for an emergency response is an active field of research and development. A preliminary theoretical consideration points out the evaluation of the benefits of geodata in emergency management and disaster scenarios as well as the requirements for the provision of geoinformation products and systems in this field of application (Fleischhauer, et al., 2017). Here, the geodata is definitively involved in all phases and scenarios of emergency management processes from prevention to immediate reaction. Currently, the techniques for geodata collection are being rapidly developed, like UAV based data recording with high resolution cameras, laser scanner technique, etc. (Colomina \& Molina, 2014). At the same time, the tools for geo-data management have been also developed over the last years. For example, Geographical Information System GIS can be useful in supporting an operational or situational picture in emergency situations (Hanssen, 2018).

Spatial data and GIS deliver a valuable spatial framework for reasoning about many problems that can arise in the context of emergency management. Today, a large number of EU-wide standardisation efforts are focusing on spatial data and being increasingly online accessible (Wittmann, 2017).

In emergency situations, the response phase of emergency management is the most complex phase in the entire cycle because it requires cooperation between various actors relating to emergency sectors. For this, a general conceptual approach for disaster and emergency management system has been developed based on the regulations to serve applications in Istanbul Governorship Provincial Disaster and Emergency Directorate (Ozbek, et al., 2015).

The literature review reveals that although distributed geolibraries offer numerous advantages over stand-alone geospatial databases, geodata sharing is still a well-recognized challenge with multiple facets due to the absence of a unified framework for data exchange. However, geodata sharing faces a set of high-level barriers such as: (1) data are scattered and locked within their respective sectors; (2) data are not updated and maintained regularly; (3) finding available data is relatively difficult (Sewnet, 2017). In response, we are motivated to design a proficient geodatabase concept and establish a sound geodata sharing mechanism that can help in overcoming some of the existing barriers related to geodata sharing.

\section{ANKommEn DATA CAPTURING SYSTEM}

The overall system for data recording consists of three UAVs, two UGVs (Figure 1) and a central ground and control station (Figure 2). The ground station serves as a central humanmachine interface to monitor and manage cooperative operation of the UAVs/UGVs by an operator. An exploration process of an area of interest is defined and assigned to the UAVs/UGVs based on initial geodata available like a priori known maps or ortho images. Exploration areas and tasks are updated with current information of the visual sensors such as RGB camera, IR camera and LiDAR while performing a mission. This is done by a data stream process to the ground station via data link functionalities. Within the streaming procedure, an online 2D georeferenced photo stitching as well as gathered LiDAR point clouds are transmitted. Path planning is then automatically conducted and transmitted via data links to the unmanned machines. The user interface developed by the IMN is capable to integrate multiple real-time sensor streams of all
$\mathrm{UAVs} / \mathrm{UGV}$ s to get a comprehensive overview of the scenario (Batzdorfer, et al., 2018).

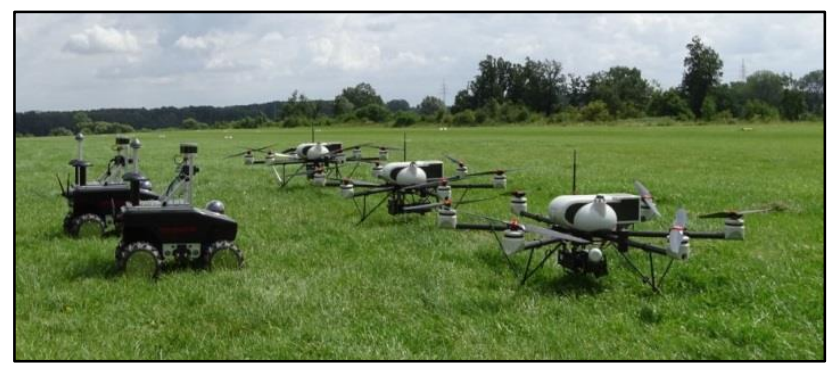

Figure 1: ANKommEn data capturing system, three UAVs Type AR200"; and two UGVs Summit XL Robotnik

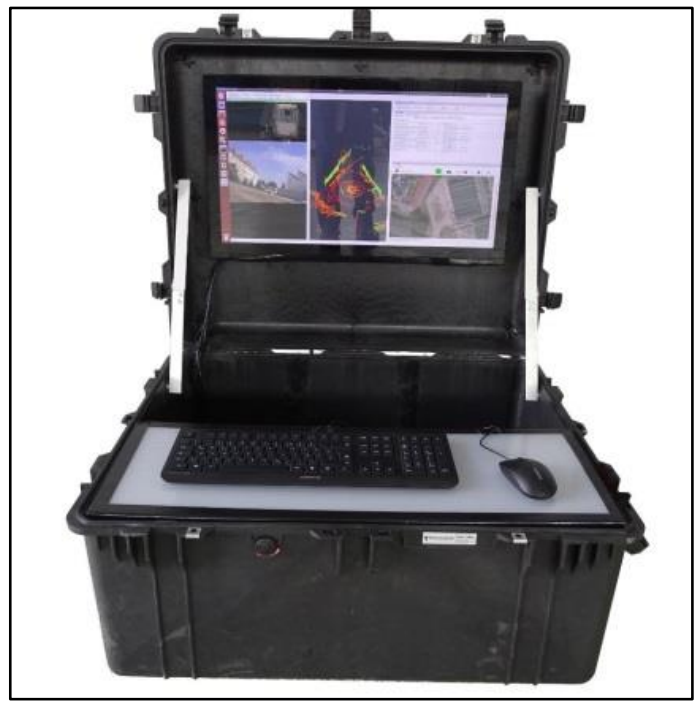

Figure 2: ANKommEn - ground control station

\section{GEODATABASE MANAGEMENT AND GEODATA DEPLOYMENT}

\subsection{Geodatabase concept}

The design of the ANKommEn geodatabase should help document, manage, and analyse geospatial data obtained. The geodatabase design can be structured into two stages (English, 2008): the conceptual and logical stages. In the conceptual phase, the data requirements should be identified and characterized. Here, involved specialists, operators and managers have to work closely with each other to define which parameters and issues in emergency management are necessary. The result of this phase produces the main geodatabase datasets. In contrast, the logical phase presents data specifications, structure, relational properties, and rules.

Within the ANKommEn2 study case, the main datasets include images and point clouds (Bobbe, et al., 2017). From a logical point of view, we classify the datasets based on data type. For example, RGB images, thermal photos, orthophotos, Digital Surface Models (DSMs), UAV's/UGV's Lidar, etc. Each type should be stored in a specific folder labelled with the corresponding ID of the actual mission (Figure 3). 


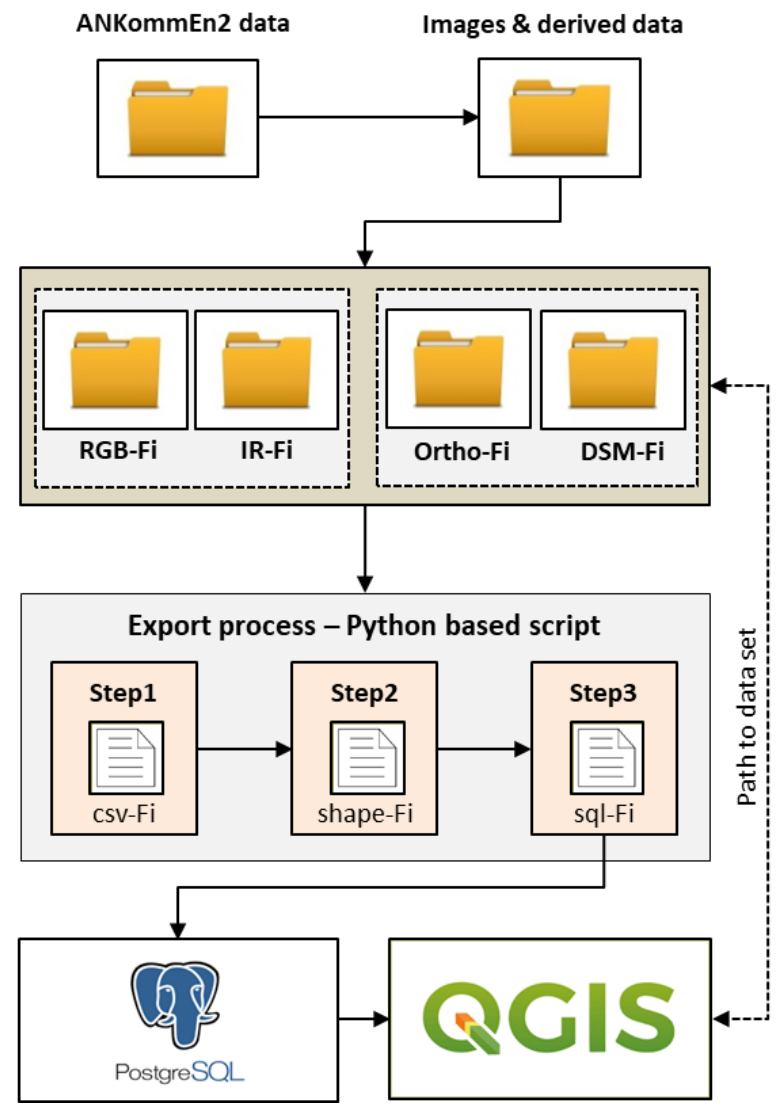

Figure 3: Storage process of images and the derived data in Postgres/PostGIS and viewing in QGIS

One key challenge in emergency management is to get the data needed quickly. Therefore, an efficient tool for data retrieval should be implemented. A suggested solution can be the Postgres $^{1}$ and the PostGIS ${ }^{2}$. The potential is that Postgres enables on the one hand a simple access to the data available and on the other hand there is an efficient compatibility with other software that deals with data processing, retrieving and viewing like QGIS, Geoserver, etc. Moreover, Postgres comes with a quite liberal licence policy. PostGIS is a spatial database extender for the Postgres object-relational database. It adds support for geographic objects allowing location queries to be run in the Structured Query Language (SQL). In Postgres/PostGIS, a dataset is stored in a spatial database allowing a smart and easy data access and retrieval. At the same time, storing images as raster layers in a PostGIS-database is complex because the Postgres/PostGIS concept does not support this issue yet. For this reason, a Python based mechanism has been achieved and developed that allows storing the geographic location of the image center points and other interested image metadata into Postgres database, and linking them to the original images. Within this mechanism, the proposed storage process is structured in three steps (Figure 3):

\section{$>$ Step 1: creation of csv-files}

The focus in this step is to infer the metadata of interest associated with images such as: the image name, image ID, the geographic coordinates of the image center points, etc. and store them in a friendly data format like csv-file. To this end, a

\footnotetext{
${ }^{1}$ https://www.postgresql.org

2 https://postgis.net/
}

Python based script has been developed to read the images captured and infer the data needed using the exif-tools automatically. At the same time, the csv-files of the images have a special syntax; here we refer to the data structure inside the csv files that enables a potential data processing and applicability for e.g. for metadata reading, conversion, etc.

\section{$>$ Step 2: csv-files to shape file format}

The csv-files created in the previous step will be converted into the shape file format. Each raw-image is represented as a point located by the image centre geographic coordinates. In addition, the absolute URL of the image file, including the IP-address of the fileserver and the data share, is stored. The benefit is to process, analyse and view the shape file data in geoinformation system software sufficiently, for e.g. in QGIS. To perform this conversion, a Python based script is used to read the csv-files and write them into shape files.

\section{Step 3: the coherence "sql-tables and shape files"}

Finally, new database tables (sql-tables) will be created based on the shape files (converted in the second step) and loaded into the target database where the sql-table should be stored. These sql-tables have a specific spatial index that enables an improved performance in terms of data archiving and retrieval.

Viewing the images of interest could be realized through a linkage between Postgres database created and QGIS. In QGIS, one can connect a specific database and select the table of interest. The Figure $(4$, a) shows a connection between both domains: QGIS and Postgres/PostGIS, namely to the database "ankommen2" and its sql-tables associated with the shape files stored in that database. The interested shape file can be imported and viewed as a layer showing the main image points (Figure 4, b, middle). Each point has its own image linkage based on the path to dataset, and therefore an image view in QGIS is possible using the "Pop-up windows" through a click event on the point of interest (Figure 4, b, right).

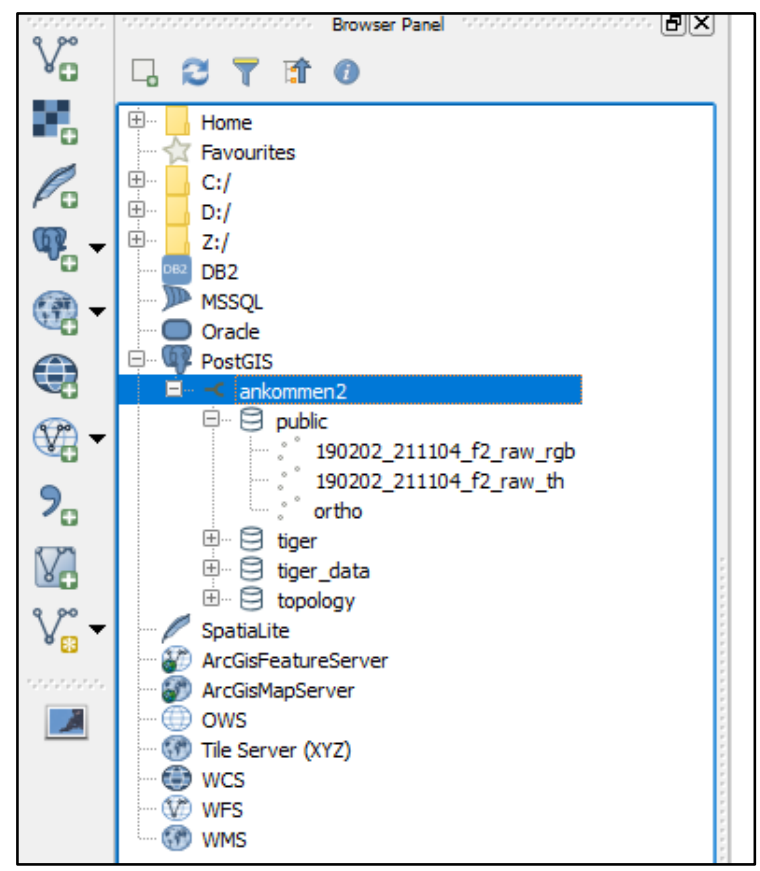

Figure (4, a): A connection between both domains: QGIS and Postgres/PostGIS 


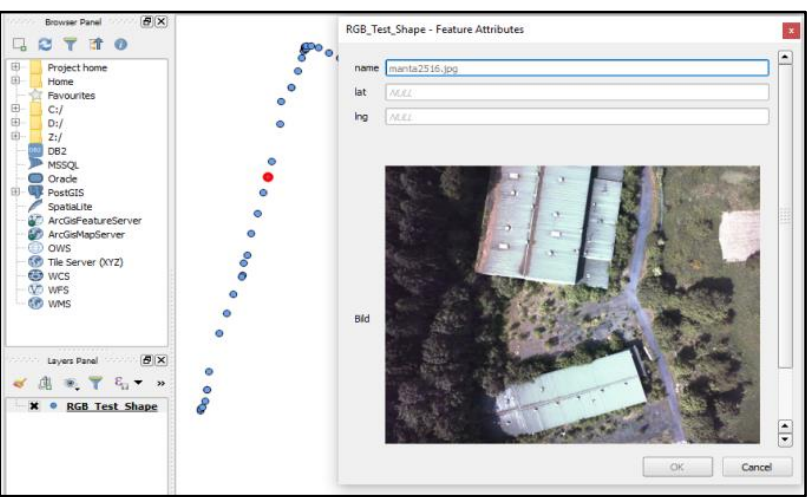

Figure $(4, \mathrm{~b})$ : Main image points as a shape layer (middle) and an image viewer with its metadata like image name, latitude, longitude, etc. (right).

At the same time, orthophotos and DSMs can be directly imported and viewed as georeferenced raster layers in QGIS independent from pop-ups functionality. The advantage is that the users and operators can edit and process the orthophotos and DSMs in an easy way.

Beside imagery datasets, ANKommEn2 data capturing system provides point clouds. On the one hand from sensors mounted on UAV/UGV systems, and on the other hand based on photogrammetric processing using RGB images. Therefore, we classify the point clouds available with respect to the sensor type used as well as labelled with flight mission ID. For e.g. UAV-point cloud, UGV-point cloud and RGB-point cloud. In the same manner for image data storage into Postgres databases, point clouds available can be also imported into a target database using a Python based script (Figure 5).

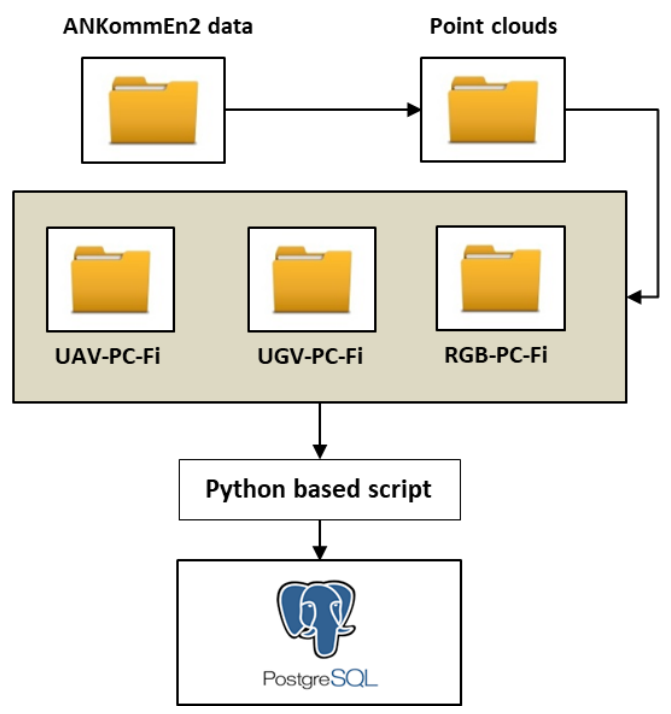

Figure 5: Storage process of point clouds in Postgres/PostGIS

\subsection{Data publishing and WMS}

The aim of an emergency manager is to provide end users with data needed in an easy way like web application that enables them to get their work done without having to learn a lot of skills or technical issues. In addition, a web-based dissemination would enable end users to even use smart phones to access the data, i.e. there is no need for specific software applications. From this point of view, we describe - in this section - a framework for geodata deployment and how to deliver the data available to end users.

Particularly, the ANKommEn2 database holds the correct and timely geospatial data and metadata that are considered the main core of the mentioned framework. Beside databases, geoweb applications need other components like: a Geoserver for data hosting, a digital basemap to provide the geographical contexts, operational layers for editing layers, queries, etc. The Figure (6) illustrates the principle of distributing geospatial information stored in ANKommEn2 databases. Here, the local geodata content (images, point clouds, etc.) is implemented into a remote web map service for end users.

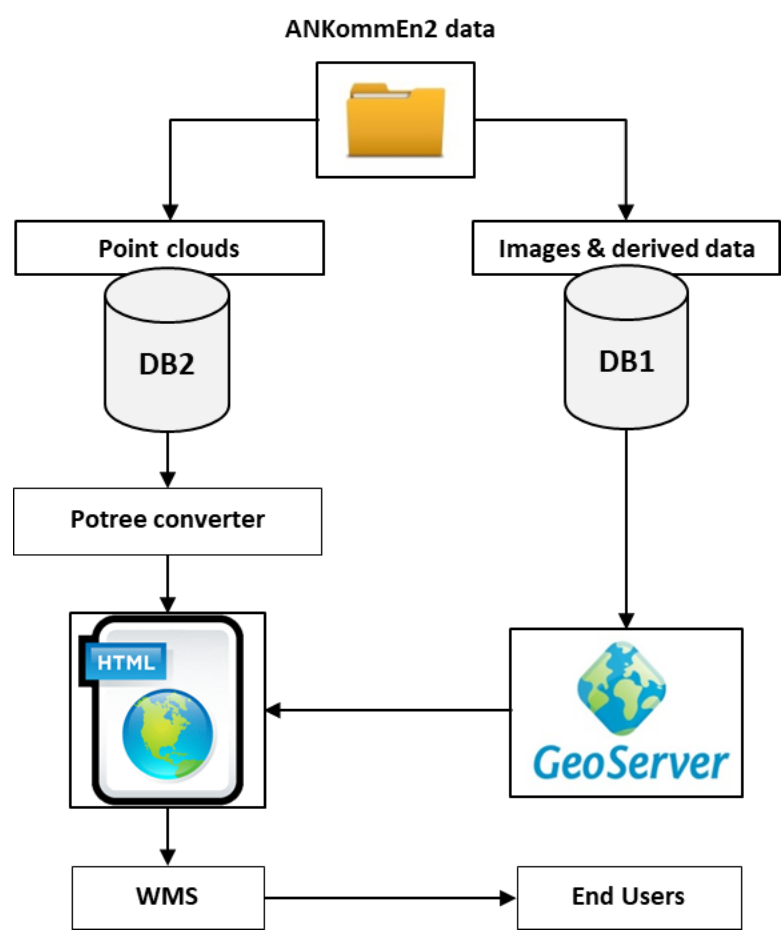

Figure 6: The concept of geo-data publishing

The images and the derived data such as orthophotos, DSMs, etc. are published based on Geoserver as georeferenced raster layers. These layers could be retrieved using their own URLs ${ }^{1}$ that to be used for the creation of friendly interactive maps. For this purpose, Leaflet is the leading open-source JavaScript library that enables creating interactive maps using $\mathrm{HTML}^{2}$ standards and $\mathrm{CCS}^{3}$. The Figure (7) shows a leaflet based web map including an orthophoto projected into the basemap "Open Street Map".

\footnotetext{
${ }^{1}$ URL: Uniform Resource Locator.

${ }^{2}$ HTML: Hypertext Markup Language Mark-up Language used for structuring and presenting content on the World Wide Web.

3 CSS: Cascading Style Sheets language used for describing the presentation of a document written in a mark-up language like HTML.
} 


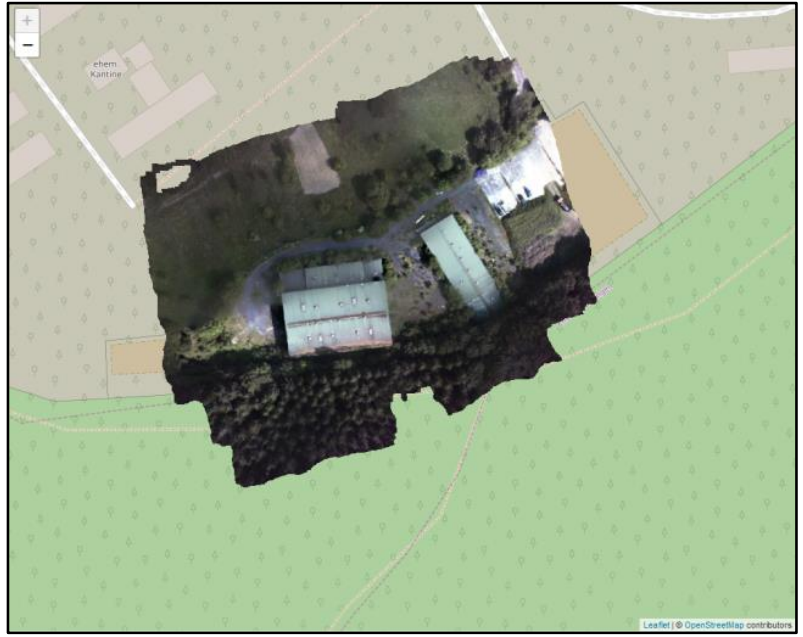

Figure 7: A leaflet based web map created for the orthophoto of the studied area. The orthophoto is published using Geoserver and defined as a layer with its own URL, which is used in leaflet based map.

In contrast, the point clouds are converted directly into the html file format using Potree Converter ${ }^{1}$ and can be retrieved as layers using their URLs (Figure 8).

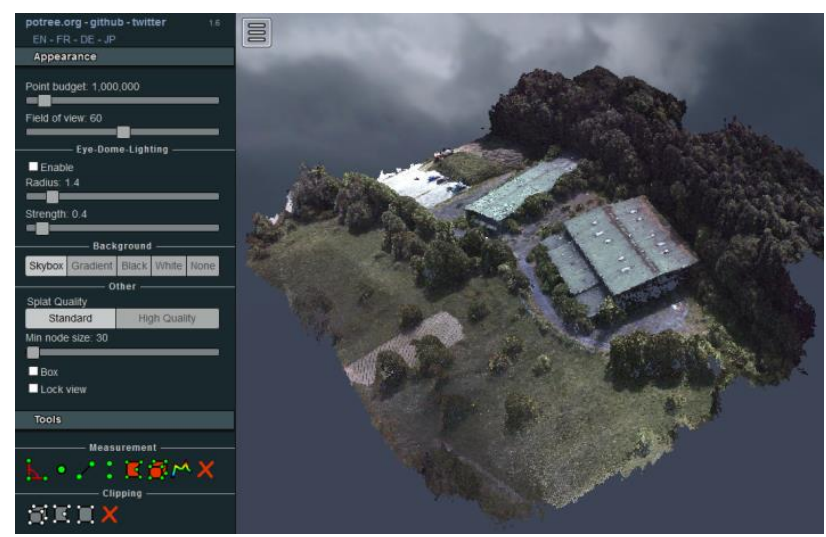

Figure 8: 3D rendering of a large point cloud of the studied area using Potree Converter Tool

Combining various data streams allows improving the information extraction, retrieving and then helping emergency managers get their work done in a best way. For this purpose, we promote to generate a so called "Hybrid Geo-Map" (HGM) based on a combined evaluation of images, point clouds, metadata, etc. The benefit expected that to keep the end users' view not more complex in getting the data requested. A leaflet based mapping allows such combination through linking data layers available. Figure (9) illustrates the initial result as a HGM applied into the leaflet-layers created of the studied area. The HGM layers created are: (a) OpenStreetMap layer, (b) the orthophoto layer and (c) the layer of point cloud. The layer of point cloud has been linked to the orthophoto using pop-up functionality (in the Figure 9, red circle, labelled with: Point Cloud-whole area, Go). By the Click event on the command

\footnotetext{
${ }^{1}$ Potree is a free open-source WebGL (Web Graphic Library) based point cloud renderer for large point clouds, developed at the Institute of Computer Graphics and Algorithms, TU Wien.
}

"Go", a new web layer will be opened including the corresponding point cloud.

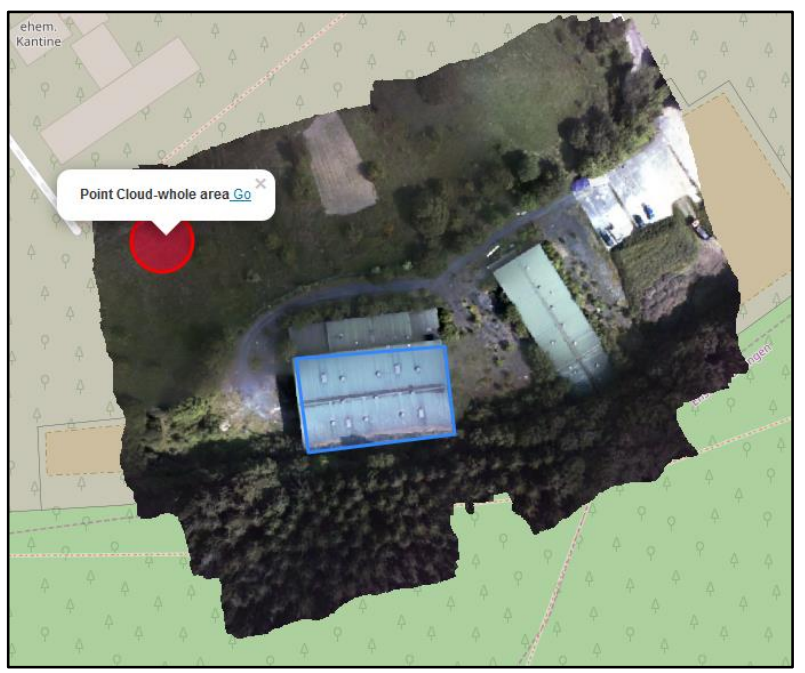

Figure 9: Hybrid Geo-Map (HGM) of the studied area

\section{POINT OF INTEREST TAGGING}

A Point of Interest (POI) is a focused geographic entity such as a landmark, building, etc. POIs are the basis for most of the data supporting location-based applications, and therefore take an important role in emergency management. POIs are stored in the database and used as geo-guiding in further applications e.g. mission planning, emergency management, etc. A reasonable process of POIs tagging, in this project, can be performed based on the HGMs created. Therefore, these HGMs have been improved in context that each object point has its own set, for example, map click event object has latitude and longitude property which is a location at which the click occurred (Figure 10). A mechanism for storing and exchanging POIs (namely, coordinates of POIs) is being developed using the most common HTTP methods "GET" and "POST". By this means end users can interact with the system and provide POIs for further consideration within the ground station.

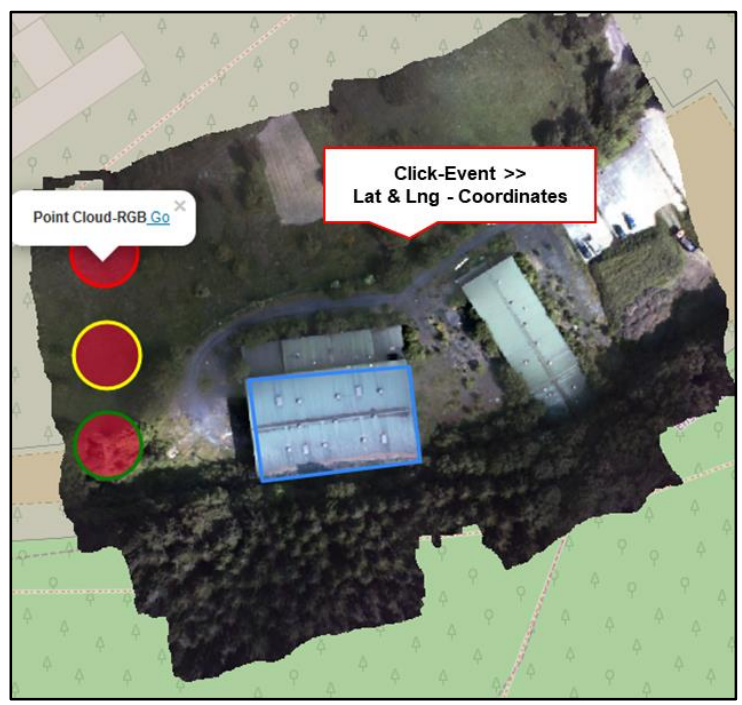

Figure 10: POIs Tagging - Latitude and longitude property of a click event object 
In the point cloud layers and based on Potree Converter Tools, measurements like object point coordinates, distances, areas and volumes can be done. The Figure (11), on the right, one can specify POIs - click event. At the same time, a storage of the measurements like: $\mathrm{X}, \mathrm{Y}$ and $\mathrm{Z}$, areas, etc. is automatically performed and easy to export for further applications like flight planning, etc.

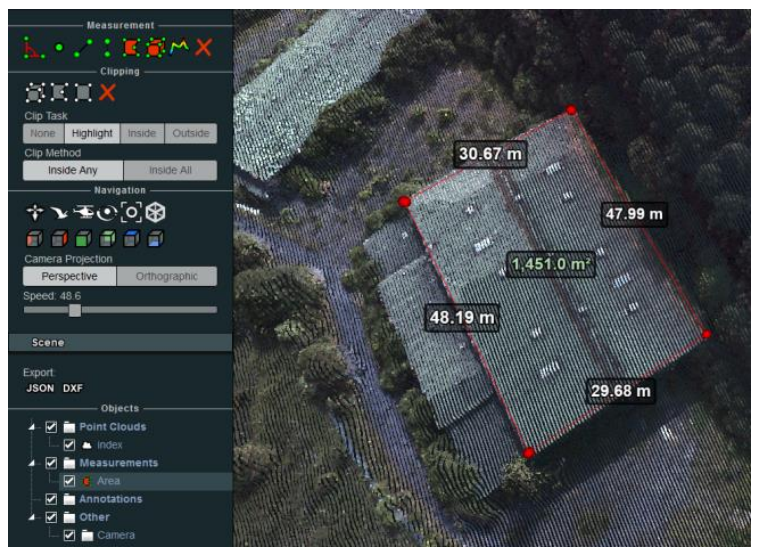

Figure 11: Measurement functionality in point cloud layer using measurement tools available in Potree Converter

\section{EVALUATION}

The challenge of a database design and dataset management draws on many different disciplines and requires tests on a large number of factors and issues like performance, data sharing, time, etc. In this regard, we address briefly in this part an assessment of the initial results and experimentations described in section 4. The assessment procedure can be expressed via an assessment matrix including, in this research project, five evaluation criteria (Table 1).

\begin{tabular}{|l|l|l|}
\hline Evaluation criteria & well & to improve \\
\hline Database design & & \\
\hline Data classification & & \\
\hline $\begin{array}{l}\text { Data storing in } \\
\text { Postgres/PostGIS }\end{array}$ & & \\
\hline $\begin{array}{l}\text { Postgres/PostGIS and } \\
\text { QGIS }\end{array}$ & & \\
\hline $\begin{array}{l}\text { Web service for data } \\
\text { provision }\end{array}$ & & \\
\hline
\end{tabular}

Table 1: Assessment matrix of the initial results

Starting with database creation, the initial results show that the database concept implemented has been well designed with respect to conceptual and logical design issues (Chapter 4.1). It enables a reasonable data processing and performance of the relevant organizational and functional tasks. Furthermore, and as expected, the data has been classified in terms of the logical definition that enables a data provision and understanding in an easy way; here it could refer on the one hand to the dataset structure and on the other hand to the intelligent IDs specified for each dataset. The IDs include a specific syntax showing the date and the time of data capturing, the data type, and the sensor/tool used for data capturing/generation. In this context, the client submits a Get-Capabilities request to the database server with date and time information, and in response, this delivers the data of interest.

Concerning on the data storage in Postgres/PostGIS, the Python based storage process should be improved to keep a high performance and time reduction in the data transmission. In addition, the connection between Postgres/PostGIS and QGIS provides a high level of data sharing and processing. This is due to the potential compatibility between both domains. In the end, the initial results reveal that the concept to create Hybrid GeoMaps based on leaflet web services is an effective and friendly tool for data provision to end users.

\section{SUMMARY}

This section summarizes the current work accomplished in the joint research project ANKommEn and its successor ANKommEn2. The main focus of this paper is to introduce an exploratory investigation to provide correct and timely geodata that can help in emergency cases; especially in support decision making in the emergency and risk management. Starting by description of the most important problems faced in emergency situations, then introducing the need for a geodatabase to assist the mangers of emergencies. In addition, a geodatabase design has been presented so that all of datasets available such as images, point clouds, etc. can be connected and retrieved. The design is diagrammed using the Postgres and its extension PostGIS. Based on WMS, the design enables end users getting their work done without having to learn a lot of skills or technical issues.

A future task is to suggest additional ideas i.e. future design considerations that can help improving the geodatabase model designed for ANKommEn2; especially in the terms of advanced relationships between geospatial data and database tables.

\section{ACKNOWLEDGEMENTS}

The presented work was done within the joint research projects ANKommEn and ANKommen2 funded by the German Federal Ministry of Economic Affairs and Energy administrated by the Space Administration of the DLR (ANKommEn - funding code: 50NA1518, ANKommEn2- funding code 50NA1807).

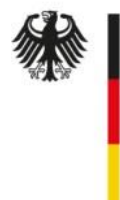

Federal Ministry

for Economic Affairs and Energy

\section{REFERENCES}

Batzdorfer, S., Bobbe, M., Becker, M., and Bestmann, U., 2018. Autonomous multi-sensor survey with teamed ground and air vehicles. In: GPS World, pp. 28-36.

Bobbe, M., Kern, A., Khedar, Y., Batzdorfer, S., and Bestmann, U., 2017. An Automated Rapid Mapping Solution Based on ORB SLAM 2 and Agisoft Photoscan API. In: Conference proceeding: IMAV 2017, Toulouse, France.

Colomina, I. and Molina, P., 2014. Unmanned aerial systems for photogrammetry and remote sensing: A review. ISPRS 
Journal of Photogrammetry and Remote Sensing 92 (2014) 79 97.

English, M., 2008. Geodatabase Design for Resource and Land Management GIS: Missoula Field Office BLM Case Study. Graduate Student Theses, Dissertations, \& Professional Papers. 487.https://scholarworks.umt.edu/etd/487.

Fleischhauer, S., Behr, F.-J. and Rawiel, P., 2017. Concept and implementation of an architecture for the immediate provision of geodata in disaster management. In: The International Archives of the Photogrammetry, Remote Sensing and Spatial Information Sciences, Volume XLII-4/W2, 2017, FOSS4GEurope 2017 - Academic Track, 18-22 July 2017, Marne La Vallée, France.

Hanssen, Ø., 2018. Position Tracking and GIS in Search and Rescue Operations. Book chapter source available at: http://dx.doi.org/10.5772/intechopen.75371.

Ozbek, E. D., Ates, and S., Aydinoglu, A.C., 2015. Using geodata corporately on the response phase of emergency management. In: The International Archives of the Photogrammetry, Remote Sensing and Spatial Information Sciences, Volume XL-3/W3, 2015, ISPRS Geospatial Week 2015, 28 Sep - 03 Oct 2015, La Grande Motte, France.

Sewnet, G. H., 2017. Geospatial Data Sharing Barriers across Organisations and the Possible Solution for Ethiopia. International Journal of Spatial Data Infrastructures Research, 2017, Vol.12, 62-84.

Wittmann, H., 2017. Value of Spatial information for Emergency Response Organisations. EENA Operations Document, Version 2 (16-01-2017). 